A disagreement-percolation type uniqueness condition for Gibbs states in models with longrange interactions

This content has been downloaded from IOPscience. Please scroll down to see the full text.

J. Stat. Mech. (2014) P10014

(http://iopscience.iop.org/1742-5468/2014/10/P10014)

View the table of contents for this issue, or go to the journal homepage for more

Download details:

IP Address: 139.179.2.116

This content was downloaded on 05/06/2015 at $11: 53$

Please note that terms and conditions apply. 


\title{
A disagreement-percolation type uniqueness condition for Gibbs states in models with long-range interactions
}

\author{
Azer Kerimov \\ E-mail: kerimov@fen.bilkent.edu.tr \\ Received 28 July 2014 \\ Accepted for publication 23 August 2014 \\ Published 9 October 2014 \\ Online at stacks.iop.org/JSTAT/2014/P10014 \\ doi:10.1088/1742-5468/2014/10/P10014
}

Bilkent University, Department of Mathematics, 06800 Bilkent, Ankara, Turkey

Abstract. We extend a condition for the uniqueness of Gibbs states in terms of percolation in the coupling of two independent realizations to lattice spin models with long-range interactions and a not necessarily unique ground state.

Keywords: rigorous results in statistical mechanics, classical phase transitions (theory), percolation problems (theory) 


\section{Contents}

1. Introduction 2

2. The main result 2

3. Applications and final remarks 5

$\begin{array}{ll}\text { References } & 6\end{array}$

\section{Introduction}

There are several different approaches to the problem of the uniqueness of Gibbs states. The most vigorous and comprehensive is the method of cluster expansions, where the local characteristics of a field are depicted in terms of clusters. Unfortunately, this method needs a small parameter and works only in the low-temperature ferromagnetic or high temperature regions, in the region of large magnetization, etc. The concept of cluster expansions goes back to [1]. Later on, the rigorous mathematical theory of cluster expansions was developed in [2-11]. The other known theory called the 'Dubrushin uniqueness method', which is based on a kind of contraction argument and which is the method of estimating the overall interaction of a spin with all other spins [12]. There is also a unique condition applicable only to $1 \mathrm{D}$ models, which requires the finiteness of the total interaction energy of the spin on any two complementary half-lines [13-17]. An alternative method for establishing the absence of phase transition reduces the uniqueness problem to that of the percolation of special clusters [18]. This method is especially powerful in 1D models with very slowly decreasing potentials when the classical methods mentioned above fail to work. The origin of the main idea of this method goes back to [19], where the uniqueness theorem for 1D antiferromagnetic models was established. An apparent deficiency of this method is the fact that it works only in models with a unique ground state. In the present paper we generalize the method by eliminating the condition of ground state uniqueness.

\section{The main result}

Let $G$ be connected, a countable infinite and locally finite graph. We assume that the spin variables $\phi(x) \in \Phi$, where $\Phi$ is a finite set. Let:

$$
H_{0}(\phi)=\sum_{<x, y>} J(\phi(x), \phi(y))
$$

where the summation is taken over all the nearest neighbors $\langle x, y\rangle ; x, y \in G$, and $J(\phi(x), \phi(y))$ is a translationally invariant function. 
A disagreement-percolation type uniqueness condition for Gibbs states in models with long-range interactions

Consider a model on $G$ with the formal Hamiltonian:

$$
H(\phi)=H_{0}(\phi)+\sum_{B \subset G} U(\phi(B))
$$

where $\phi(B)$ denotes the restriction of the configuration $\phi$ to the set $B$, and the potential $U(\phi(B))$ is a translationally invariant function. On the potential $U(\phi(B))$ we impose a natural condition, necessary for the existence of the thermodynamic limit:

$$
\sum_{B \subset G: x \in B}|U(\phi(B))|<\mathrm{const}
$$

where the const does not depend on the configuration $\phi$.

Let $V$ be a finite volume in $G$. The concatenation of the configurations $\phi(V)$ and $\phi^{i}$ we denote by $\phi \diamond \phi^{i}: \phi \diamond \phi^{i}(x)=\phi(x)$ if $x \in V$, and $\phi \diamond \phi^{i}(x)=\phi^{i}(x)$ if $x \in G-V$. The finite-volume of the Gibbs distribution corresponding to the boundary conditions $\phi^{i}$ is:

$$
\mathbf{P}_{\mathbf{V}}^{\mathbf{i}}\left(\phi \mid \phi^{i}\right)=\frac{\exp \left(-\beta\left(H\left(\phi \diamond \phi^{i}\right)-H\left(\phi^{i}\right)\right)\right)}{\Xi\left(V, \phi^{i}\right)}
$$

where $\beta$ is the inverse temperature and the partition function $\Xi\left(V, \phi^{i}\right)=$ $\sum_{\phi \in V} \exp \left(-\beta H_{V}\left(\phi \diamond \phi^{i}\right)-H\left(\phi^{i}\right)\right)$. A probability measure $\mathbf{P}$ on the configuration space $\Phi^{G}$ is said to be an infinite-volume Gibbs measure if for each $V$ and for $\mathbf{P}$ almost all $\phi^{i}$ in $\Phi^{G}$ we have:

$$
\mathbf{P}\left(\phi(V) \mid \phi^{i}(G-V)=\mathbf{P}_{\mathbf{V}}^{\mathbf{i}}\left(\varphi \mid \phi^{i}\right)\right.
$$

where $\mathbf{P}\left(\phi(V) \mid \phi^{i}(G-V)\right.$ is a conditional probability of $\phi(V)$ given $\phi^{i}(G-V)$.

For each $A \subset G$ the boundary $\delta(A)$ of $A$ is the set of all the points not belonging to $A$ but incident to $A$. Let $\phi$ be a fixed configuration:

$$
\begin{aligned}
\exp (-\beta H(\phi)) & =\exp \left(-\beta H_{0}(\phi)\right) \exp \left(-\beta \sum_{B \subset G} U(\phi(B))\right) \\
& =\exp \left(-\beta H_{0}(\phi)\right) \prod_{B \subset G} \exp (-\beta U(\phi(B))) \\
& =\exp \left(-\beta H_{0}(\phi)\right) \prod_{B \subset G}(1+\exp (-\beta U(\phi(B))-1) \\
& =\exp \left(-\beta H_{0}(\phi)\right) \prod_{B \subset G}\left(1+\gamma_{B}\right)
\end{aligned}
$$

where $\gamma_{B}=\exp (-\beta U(\phi(B)))-1$. Now, by expanding (4) we get:

$$
\exp (-\beta H(\phi))=\exp \left(-\beta H_{0}(\phi)\right) \sum_{q \subset M} \prod \gamma_{B}
$$

where $M$ is the set of all $B \subset G$ except pairs of the nearest neighboring points. In other words, instead of one configuration $\phi$ with an interaction of $H(\phi)$ and a statistical weight of $\exp (-\beta H(\phi))$ we get infinitely many copies $\phi_{q}$ of the configuration (each copy $\phi_{q}$ is equipped with different sub-interactions $q$, and each $q$ is the union of some subsets $B$ ) each with a statistical weight of $\exp \left(-\beta H_{0}(\phi)\right) \prod_{B \in q} \gamma_{B}$. The main point in this new 
A disagreement-percolation type uniqueness condition for Gibbs states in models with long-range interactions

representation is that if in any sub interaction-equipped configuration two points are not connected by an interaction element, then the two points are not interacting: We have 'got rid of' long-range interactions [18] and the Markov property holds: If we define Gibbs measures by using the weights (5) as in (3), then $\mathbf{P}\left(\phi_{q}(V) \mid \phi^{i}(G-V)\right.$ only depends on $\phi^{i}(\delta V \cup q)$.

Suppose that $\phi_{p}^{\prime}$ and $\phi_{q}^{\prime \prime}$ are two configurations equipped with the sub-interactions $p$ and $q$, respectively. Let $D \subset G$ be the set of all points $x \in G$ on which configurations $\phi_{q_{1}}^{\prime}$ and $\phi_{q_{2}}^{\prime \prime}$ disagree: $\phi_{p}^{\prime} \neq \phi_{q}^{\prime \prime}$ Two points $x, y \in D$ are adjacent if $\operatorname{dist}(x, y)=1$. Two points $x, y \in D$ are interaction connected if $x, y \in B \in p$, or $x, y \in B \in q$. A path from $\bar{x} \in D$ to $\bar{y} \in D$ is a sequence of vertices $x_{1}=\bar{x}, x_{2}, \ldots, x_{l}=\bar{y}$, where for each $i=1,2, \ldots, l-1$ the points $x_{i}$ and $x_{i+1}$ are adjacent or interaction connected. We say that the set of points $D^{*} \subset D$ is a path of disagreement, if any two points $x, y \in D^{*}$ there is a path from $x$ to $y$.

Let $\mu_{1}$ and $\mu_{2}$ be two Gibbs measures for the same interaction.

Theorem 1. Suppose that the $\left(\mu_{1} \times \mu_{2}\right)\left(\phi_{p}^{\prime}, \phi_{q}^{\prime \prime}\right)$ probability of the event is an infinite path of disagreement corresponding to zero. Then $\mu_{1}=\mu_{2}$.

Proof. Let $A$ be an arbitrary finite set of vertices and $\phi(A)$ be any configuration on $A$. In order to prove the theorem we have to prove that $\mu_{1}(\phi(A))=\mu_{2}(\phi(A))$. For each pair $\left(\phi_{p}^{\prime}, \phi_{q}^{\prime \prime}\right)$ the cluster of disagreement $C_{A}=C\left(A, \phi^{\prime}, \phi^{\prime \prime}\right)$ containing $A$ is the set $A$ and all lattice points $x \in \mathbf{Z}^{\nu}$ for which there exists a path of disagreement connecting $x$ and $A \cup \delta(A)$. Let $T: \Omega \times \Omega \rightarrow \Omega \times \Omega$ be the transformation which exchanges $\phi^{\prime}$ and $\phi^{\prime \prime}$ on $C_{A}: T\left(\phi_{p}^{\prime}, \phi_{q}^{\prime \prime}\right)=\left(\psi_{p}^{\prime}, \psi_{q}^{\prime \prime}\right)$ where

$$
\begin{aligned}
& \psi_{p}^{\prime}(x)= \begin{cases}\phi_{p}^{\prime \prime}(x) & \text { if } x \in C_{A} \\
\phi_{p}^{\prime}(x) & \text { otherwise }\end{cases} \\
& \psi_{q}^{\prime \prime}(x)= \begin{cases}\phi_{q}^{\prime}(x) & \text { if } x \in C_{A} \\
\phi_{q}^{\prime \prime}(x) & \text { otherwise }\end{cases}
\end{aligned}
$$

The transformation $T$ is readily one-to-one. If there is no infinite disagreement cluster for pair $\left(\phi^{\prime}, \phi^{\prime \prime}\right)$ then by Markov the property of Gibbs measures

$$
\left(\mu_{1} \times \mu_{2}\right)\left(\phi_{p}^{\prime}, \phi_{q}^{\prime \prime}\right)=\left(\mu_{1} \times \mu_{2}\right)\left(\psi_{p}^{\prime}, \psi_{q}^{\prime \prime}\right) .
$$

Therefore, since $C_{A}$ is finite with probability one, (5) is held with probability one. Finally, by (5):

$$
\begin{aligned}
\mu_{1}(\phi(A)) & =\sum_{\left(\phi_{p}^{\prime}, \phi_{q}^{\prime \prime}\right): \phi_{p}^{\prime}(A)=\phi(A)}\left(\mu_{1} \times \mu_{2}\right)\left(\phi_{p}^{\prime} \times \phi_{q}^{\prime \prime}\right) \\
& \left.=\sum_{\left(\psi_{p}^{\prime}, \psi_{q}^{\prime \prime}\right): \psi_{q}^{\prime \prime}(A)=\phi(A)}\left(\mu_{1} \times \mu_{2}\right)\left(\psi_{p}^{\prime} \times \psi_{q}^{\prime \prime}\right)\right)=\mu_{2}(\phi(A))
\end{aligned}
$$

where the summation in $\sum_{\left(\psi_{p}^{\prime}, \psi_{q}^{\prime \prime}\right): \psi_{q}^{\prime \prime}(A)=\phi(A)}$ is taken over by all the pairs of equipped configurations $\left(\phi_{p}^{\prime}, \phi_{q}^{\prime \prime}\right)$ such that there is no infinite path of disagreement connected to $A$ and $\phi_{p}^{\prime}(A)=\phi(A)$; the summation in $\sum_{\left(\psi_{p}^{\prime}, \psi_{q}^{\prime \prime}\right): \psi_{q}^{\prime \prime}(A)=\phi(A)}$ takes over all the pairs of the equipped configurations $\left(\psi_{p}^{\prime}, \psi_{q}^{\prime \prime}\right)$ such that there is no infinite path of disagreement connected to $A$ and $\psi_{q}^{\prime \prime}(A)=\phi(A)$. The proof is complete. 
A disagreement-percolation type uniqueness condition for Gibbs states in models with long-range interactions

\section{Applications and final remarks}

Now we apply the disagreement-percolation criterion to the ferromagnetic Ising model with long-range interaction on $Z^{2}$ with the following Hamiltonian:

$$
H(\phi)=-\sum_{x, y \in Z^{2}, \operatorname{dist}(x, y)=1} \phi(x) \phi(y)-\sum_{x, y \in Z^{2}, \operatorname{dist}(x, y)>1} c^{-\operatorname{dist}(x, y)} \phi(x) \phi(y)=H_{0}+H_{1}
$$

Theorem 2. There are positive constants $\beta_{0}$ and $c_{0}$ as for all non-negative $\beta<\beta_{0}$ and $c<c_{0}$ the model (7) has a unique limiting Gibbs state.

Proof. The uniqueness of Gibbs states will be proved by employing theorem 1. Let $\mu_{1}$ and $\mu_{2}$ be two Gibbs measures corresponding to the Hamiltonian (7). In order to do this we will prove that the $\left(\mu_{1} \times \mu_{2}\right)$ probability of the event is an infinite path of zero disagreement. Let $\mathbf{P}_{\mathbf{V}}^{\mathbf{i}}\left(\varphi \mid \phi^{i}\right)$ be the finite volume Gibbs distribution corresponding to the Hamiltonian $H_{0}$, given a finite volume $V \subset Z^{2}$ and boundary conditions $\phi^{i}$. Let $x^{\prime} \in V$. Since the model (7) is ferromagnetic there is a $\beta_{1}$, such that for all $\beta<\beta_{1}$ :

$$
\mathbf{P}_{\mathbf{V}}^{\mathbf{i}}\left(\phi\left(x^{\prime}\right)=1 \mid \phi^{i}\right) \leqslant \frac{\mathrm{e}^{4 \beta}}{\mathrm{e}^{4 \beta}+\mathrm{e}^{-4 \beta}}<0.501
$$

The first inequality in (8) is due to Griffiths inequality (the probability of +1 is maximal when all other spins are +1$)$. The second inequality is due to the fact that $\frac{\mathrm{e}^{4 \beta}}{\mathrm{e}^{4 \beta}+\mathrm{e}^{-4 \beta}}=\frac{1}{2}$ at $\beta=0$ and the dependence on $\beta$ is continuous. A similar inequality holds for the probability of $\mathbf{P}_{\mathbf{V}}^{\mathbf{i}}\left(\phi\left(x^{\prime}\right)=-1 \mid \phi^{i}\right)$. Since the interaction in the model (7) is pairwise the interaction elements are $\gamma_{B}=\gamma(x, y)=\exp \left( \pm \beta c^{-\operatorname{dist}(x, y)}\right)-1$. There is $\beta_{2}$, so that for $\beta<\beta_{2}$ we have $\gamma(x, y) \leqslant c^{-\operatorname{dist}(x, y)}$. We choose $\beta_{0}=\min \left(\beta_{1}, \beta_{2}\right)$. If two points of disagreement are connected by some interaction element we will assume that these two points are connected through the shortest path of the neighboring lattice points. All the lattice points lying on the path will be called interaction points. If the path connects points $x^{\prime}=\left(a^{\prime}, b^{\prime}\right)$ and $y^{\prime}=\left(a^{\prime \prime}, b^{\prime \prime}\right)$ and $\left|a^{\prime}-a^{\prime \prime}\right|=t_{1}$ and $\left|b^{\prime}-b^{\prime \prime}\right|=t_{2}$, then by arithmeticquadratic mean inequality $t_{1}+t_{2} \leqslant 2 \sqrt{t_{1}^{2}+t_{2}^{2}}=\sqrt{2}|\rho|$. By a uniform 'distribution' of the weight $c^{-\operatorname{dist}(x, y)}$ to all $t=t_{1}+t_{2}$ interaction points we get $\gamma(x, y) \leqslant(\sqrt[\sqrt{2}]{c})^{t}$. This means that the statistical weight of the interaction between the two points over the interaction path is the product of the weights of the interaction points, each not exceeding $\sqrt[\sqrt[2]{2}]{c}$. Thus, by Peierls' argument the probability that a given lattice point is an interaction point is also at most $\sqrt[\sqrt{2}]{c}$ (let $x$ be a lattice point, to each configuration equipped with sub-interactions for which $x$ is an interaction point in the numerator we correspond a configuration equipped with sub-interactions for which $x$ is not an interaction point in the denominator). Since we are considering a pair of configurations, the probability that a lattice point is an interaction point is at most $1-\left(1-c^{\frac{1}{\sqrt{2}}}\right)^{2}$. Choosing a $c_{0}$ such that for all $c<c_{0}$ we have $1-\left(1-c^{\frac{1}{\sqrt{2}}}\right)^{2}<0.05$. Now we are ready to estimate the disagreement paths. By (8) the probability that the lattice point $x$ is a point of disagreement is at most $2 \cdot 0.501 \cdot 0.501<0.5021$. Since the path of disagreement consists of disagreement lattice points and possible interaction points then the probability of an infinite path of disagreement is no greater than the probability of site percolation with $p=0.5021+0.05=0.5521$. 
A disagreement-percolation type uniqueness condition for Gibbs states in models with long-range interactions

Since the site percolation threshold for $Z^{2}$ exceeds 0.556 (see [23]) the probability of infinite disagreement-percolation is zero and by theorem 1 we are done.

Theorem 1 is proved by the method of coupling using Gibbs measures. Different methods involving coupling ideas were previously [20-22]. The term 'disagreement' we adopt from [21] and [22]. The similar disagreement uniqueness condition for models with an interaction of neighboring spins was firstly formulated in [21]. The crucial transformation of $T\left(\phi^{\prime}, \phi^{\prime \prime}\right)=\left(\psi^{\prime}, \psi^{\prime \prime}\right)$ in the proof of theorem 1 is also taken from [21]. In [19] the uniqueness of Gibbs states in 1D with long-range antiferromagnetic Ising models has been proved again by the method of coupling two independent realizations. Afterwards this method was generalized for models with a unique ground state [18]. The method employed in $[18,19]$ also uses the finite-volume version of the transformation $T\left(\phi^{\prime}, \phi^{\prime \prime}\right)=\left(\psi^{\prime}, \psi^{\prime \prime}\right)$. In $[18,19]$ the undesirable products of coupling are clusters connecting the set $A$ with the boundary conditions. When these clusters are negligible, the two Gibbs states become completely continuous with respect to each other and coincide. Since the models in $[18,19]$ have a unique ground state, the disagreement clusters are defined as clusters not coinciding with a unique ground state. Thus, theorem 1 generalizes theorem 1 of [18] for models with long-range interaction (in a case when only neighboring spin variables interact, theorem 1 becomes theorem 1 of [21]) and generalizes theorem 2 of [18] for models having more then one ground state. That is why in this section we have applied the disagreement-percolation uniqueness criterion (theorem 1) to models (7) having more than one ground state.

\section{References}

[1] Mayer J E 1947 Integral equations between distribution functions of molecules J. Chem. Phys. 15187

[2] Minlos R A and Sinai Y G 1967 The phenomenon of 'separation of phases' at low temperatures in certain lattice models of a gas I Mat. Sb. 73375

[3] Minlos R A, Sinai Y G 1968 The phenomenon of 'separation of phases' at low temperatures in certain lattice models of a gas II Trans. Moscow Math. Soc. 19113

[4] Ruelle D 1969 Statistical Mechanics (New York: Benjamin)

[5] Groeneveld J 1967 Estimation methods for Mayer's graphical expansions I, II, III Nederl. Akad. Wetensch. Proc. B 70 454, 468, 475

[6] Gruber C and Kunz H 1971 General properties of polymer systems Commun. Math. Phys. 22133

[7] Penrose O 1963 Convergence of fugacity expansions for fluids and lattice gases J. Math. Phys. 41312

[8] Penrose O 1963 The remainder in Mayer's fugacity series J. Math. Phys. 41488

[9] Pirogov S A and Sinai Y G 1975 Phase diagrams of classical lattice systems Teor. Math. Phys. 25358

[10] Pirogov S A and Sinai Y G 1976 Phase diagrams of classical lattice systems Teor. Math. Phys. 2661

[11] Malyshev V A 1980 Cluster expansions in lattice models of statistical physics and quantum field theory Russ. Math. Surveys 2212

[12] Dobrushin R L 1968 Description of a random field by means of conditional probabilities and conditions for its regularity Theor. Prob. Appl. 13201

[13] Ruelle D 1968 Statistical mechanics of a 1D lattice gas Commun. Math. Phys. 9267

[14] Dobrushin R L 1968 Gibbsian random fields for lattice systems with pairwise interactions Funct. Anal. Appl. 231

[15] Dobrushin R L 1969 Gibbsian random fields. General case Funct. Anal. Appl. 327

[16] Bricmont J, Lebowitz J L and Pfister C E 1979 On the equivalence of boundary conditions J. Stat. Phys. 21573 


\section{A disagreement-percolation type uniqueness condition for Gibbs states in models with long-range interactions}

[17] Cassandro M and Olivieri E 1981 Renormalization group and analyticity in one dimension: a proof of Dobrushin's theorem Commun. Math. Phys. 80255

[18] Kerimov A 2002 The relationship between phase transitions and percolation in models with ground-state degeneracy J. Phys. A: Math. Gen. 355365

[19] Kerimov A 1993 Absence of phase transitions in 1D antiferromagnetic models with long-range interactions J. Stat. Phys. 72571

[20] Maes C 1993 Coupling interacting particle systems Rev. Math. Phys. 5457

[21] van den Berg J 1993 A uniqueness condition for Gibbs measures, with application to the 2D Ising antiferromagnet Commun. Math. Phys. 152161

[22] van den Berg J and Maes C 1994 Disagreement percolation in the study of Markov fields Ann. Prob. 22749

[23] van den Berg J and Ermakov A A 1996 A new lower bound for the critical probability of site percolation on the square lattice Random Struct. Algorithms 8199 